\title{
Dominique-Marie Varlet, missionnaire en Nouvelle-France (1713-1718)
}

\section{Pierre Hurtubise}

Volume 35, 1968

URI : https://id.erudit.org/iderudit/1007304ar

DOI : https://doi.org/10.7202/1007304ar

Aller au sommaire du numéro

Éditeur(s)

Les Éditions Historia Ecclesiæ Catholicæ Canadensis Inc.

ISSN

0318-6172 (imprimé)

1927-7067 (numérique)

Découvrir la revue

Citer cet article

Hurtubise, P. (1968). Dominique-Marie Varlet, missionnaire en Nouvelle-France (1713-1718). Sessions d'étude - Société canadienne d'histoire de l'Église catholique, 35, 21-32. https://doi.org/10.7202/1007304ar

Tous droits réservés @ Les Éditions Historia Ecclesiæ Catholicæ Canadensis Inc., 1968
Ce document est protégé par la loi sur le droit d'auteur. L'utilisation des services d'Érudit (y compris la reproduction) est assujettie à sa politique d'utilisation que vous pouvez consulter en ligne.

https://apropos.erudit.org/fr/usagers/politique-dutilisation/ 


\section{Dominique-Marie Varlet, missionnaire en Nouvelle-France (1713-1718)}

La sainte horreur et l'espèce de répulsion qu'inspirait autrefois chez nous - et ailleurs - la simple mention du mot jansénisme ont conduit nombre de nos historiens ecclésiastiques à chercher à minimiser le rôle joué en Nouvelle-France par Dominique-Marie Varlet (1678-1742), futur appelant et futur père spirituel de l'Église schismatique d'Utrecht ${ }^{1}$, quand elles n'ont pas valu à ce dernier l'honneur d'un silence que l'on pourrait difficilement qualifier de respectueux ${ }^{2}$. Sans doute Varlet ne fit-il qu'un assez bref séjour en Amérique - six années au total et son zèle apostolique ne trouva-t-il à s'exercer que dans des régions, à vrai dire, fort éloignées de ce qui constituait déjà, à l'époque, le Canada, mais les relations, si épisodiques fussent-elles, qu'il se devait d'entretenir avec l'évêque de Québec, son supérieur immédiat, puis, surtout, le séjour de près de treize mois qu'il fit dans la ville épiscopale au cours des années 1717-1718 nous incitent à penser que son influence en terre canadienne ne fut pas si négligeable ni si éphémère que certains ont voulu ou voudraient parfois nous le faire croire. Certes DominiqueMarie Varlet ne saurait être considéré comme une des figures marquantes de notre histoire religieuse au $\mathrm{XVIII}^{\circ}$ siècle, mais ne serait-ce que pour corriger un certain nombre d'inexactitudes, voire d'erreurs manifestes accumulées sur son compte, il n'est peut-être pas inutile que nous ouvrions une fois de plus son dossier, d'une part, pour y verser un certain nombre de pièces nouvelles, d'autre part, pour le situer dans un cadre historique beaucoup plus vaste, avec l'espoir d'en tirer à la

1 Un certain nombre de notices biographiques ont été consacrées à Varlet. Citons, en particulier, L. MoRERI, Le grand dictionnaire historique, éd. 1759, t. X, p. 481; J. F. Michaud, Biographie universelle, t. XLII, p. 649; E. AmanN, D.T.C., t. XV, col. 2535-2536. Toutes, cependant, s'inspirent d'une seule et même source: la notice nécrologique consacrée à Varlet par les Nouvelles Ecclésiastiques, année 1742 , pp. 105-108. C'est cette dernière que nous utilisons plus particulièrement ici. Pour tout ce qui concerne son séjour en Amérique, les renseignements fournis par Anselme RhÉAUMe (Mgr Dominique-Marie Varlet, dans Bull. Rech. hist., III (1897), pp. 18-22) et Auguste Gosselin (L'Eglise du Canada, t. I, Québec 1911, pp. 331-335) doivent être corrigés par ceux que Maximin Deloche a tiré de la correspondance même de Varlet : Un missionnaire français en Amérique au XVIII' siècle. Contribution à l'histoire de l'établissement des Français en Louisiane, dans Bull. de la sect. de géogr. du Comité des travaux historiques, Paris, 1930 [1931], t. XLV, pp. 39-60.

2 Ânsi A. Launay, dans son Histoire générale de la Société des MissionsEtrangères (t. I, Paris 1894), a-t-il trouvé le moyen de taire le nom de Varlet là même où il était presque impossible de le faire, par exemple dans un chapitre consacré aux missions de la Société en Nouvelle-France au début du XVIII ${ }^{\circ}$ siècle (p. 441 et suiv.) et dans un passage concernant le jansénisme (p. 491 et suiv.). 
fin une image plus exacte et beaucoup plus conforme à la réalité de cet homme étrange, voire contestable, mais généreux qui n'hésita pas à franchir les mers en $\mathrm{I} 7 \mathrm{I} 3$ pour venir en Amérique se consacrer à l'évangélisation d'âmes qu'il considérait parmi les plus abandonnées.

\section{L'homme et son milieu}

Né à Paris, le 15 mars 1678, d'une famille probablement originaire de Picardie, mais installée dans la capitale denuis au moins une trentaine d'années - son grand-père, " noble homme * Hector Varlet, capitaine du château de Nanteuil, s'était marié à Saint-Germain-l'Auxerrois en 1634 - Dominique-Marie Varlet avait été baigné, dès sa plus tendre enfance, dans un climat qui, contrairement à ce que voudraient nous faire croire, et pour cause, les Nouvelles Ecclésiastiques, ne devait pas laisser tellement présager son orientation future.

Son oncle, Charles Varlet, dit le sieur de La Grange et son propre père, Achille Varlet, alias Verneuil, appartenaient, en effet, l'un et l'autre, au monde du théâtre. Le premier était, depuis 1659, membre de la troupe du Petit-Bourbon et allait s'avérer avec les années un des plus fidèles collaborateurs de Molière. Le second, sans doute moins doué que son cadet, avait dû se contenter d'une carrière plus modeste. mais, après quelques années passées au théâtre du Marais. il n'en avait pas moins réussí, en 1673, à se faire accepter dans la troupe du Roi qui venait d'emménager au théâtre de la rue de Guénégaud. Cette même année - il avait alors 37 ans - il avait épousé une certaine "damoiselle "Valée, fille d'un chapelier parisien.

Des sept enfants issus de ce mariage, trois seulement allaient atteindre l'âge adulte: Jean-Achille, futur procureur au Parlement de Paris, Dominique-Marie, son aîné, et une fille qu'on verra plus tard épouser un procureur au Châtelet, $\mathbf{M}^{e}$ Antoine Olivier. Du monde du théâtre. on allait donc voir la famille accéder rapidement à celui de la judicature, voire même, avec le futur archevêque de Babylone, au monde d'Église. Achille Varlet n'était sans doute pas étranger à cette évolution et, eu égard à la situation dans laquelle se trouvaient les comédiens, à l'époque, il avait vraisemblablement eu à cœur de donner à ses enfants un statut social moins décrié, bien qu'il fût déjà lui-même bourgeois de Paris et qu'il pût afficher partout sans vergogne son titre, pourtant fort contestable, de sieur de Verneuil $^{3}$.

C'est ici qu'il faut situer un fait, pour le moins étonnant, rapporté par les Nouvelles Ecclésiastiques. A les en croire, Achille Varlet, chrétien

3 A moins d'indication contraire, tous les renseignements que nous donnon: ici sur la famille Varlet sont empruntés au précieux article d'A. JAL, Dictionnaire critique de biographie et d'histoire, Paris, 1867, pp. 726-729. Sur Jean-Achille Varlet et Antoine Olivier, voir également l'Almanach Royal pour les années 1715 à 1722 . 
d'une grande intériorité et d'un rare esprit d'abnégation, se serait retiré, plusieurs années avant sa mort, dans un modeste ermitage du Mont-Valérien où il aurait mené dans la plus complète solitude une vie d'ascèse et d'oraison ${ }^{4}$. Laissons de côté cette description assez improbable d'un homme qui était tout de même pensionnaire de la Comédie - il le sera jusqu'en 1706 - et attardons-nous plutôt au fait lui-même évoqué par les auteurs de la notice nécrologique de l'archevêque de Babylone.

En 1679, Verneuil avait acheté d'Armande Béjart, veuve de Molière, une maison de campagne et un jardin sis sur le grand chemin du Mont-Valérien, à peu de distance du célèbre pèlerinage dirigé par les prêtres du Calvaire ${ }^{5}$. Il n'est pas impossible qu'à un certain moment, poussé par la grâce, il ait senti le besoin d'aller y terminer ses jours dans la pénitence et le recueillement. Certes, cette dernière ambition, si tant est qu'elle exista, ne se réalisa pas jusqu'au bout. Achille Varlet étant mort en 1709 dans sa résidence de la rue de Nevers à Paris, mais cela n'exclut pas pour autant, bien qu'elle la limite jusqu'à un certain point, la possibilité d'une retraite plus ou moins prolongée du vieux comédien à Rueil. Le plus probable, toutefois, est que la famille Varlet séjourna tout simplement, à diverses reprises, dans l'ancienne maison de campagne d'Armande Béjart et qu'à partir de ce fait, le parti janséniste élabora une touchante histoire susceptible de faire oublier les véritables antécédents de l'archevêque de Babylone. Quoi qu'il en soit, cette présence des Varlet à proximité du célèbre sanctuaire du Mont-Valérien allait avoir une influence déterminante sur l'avenir du jeune Dominique-Marie.

Le Mont-Valérien, une des plus hautes collines des environs de Paris, immédiatement à l'ouest de la capitale, abritait, depuis le XVI siècle en particulier, un certain nombre d'anachorètes qui s'y livraient, dans le plus grand silence, à une vie de prière et de renoncement. En 1633 un licencié de Sorbonne, Hubert Charpentier, avait obtenu du roi la permission de planter sur le sommet de la colline trois croix représentant le mont du Calvaire, d'y élever une église en l'honneur de la Sainte-Croix et d'y construire une maison destinée aux prêtres chargés de desservir le nouveau pèlerinage appelé, selon le vœu de son fondateur, à attirer de grands concours de dévots aussi bien de Paris que des environs ${ }^{6}$.

Les ermites, pour leur part, avaient fini par se former en communauté - ils seront une dizaine en 1715 - tandis que les prêtres desservants,

4 Nouvelles Ecclésiastiques, loc. cit., p. 105.

5 Michel Gurlsot, c La famille de Molière à Suresnes ", dans Bulletin de la Société historique de Suresnes, V (1965), pp. 230-232.

6 A. M. LEFÈvre, Calendrier historique et chronologịnue de l'Eglice de Paris, Paris 1747, pp. 391-396. 
connus sous le nom de Prêtres du Calvaire, après une période d'incertitude et de tâtonnements dans la seconde moitié du XVII ${ }^{e}$ siècle, avaient réussí à se regrouper, voire mème à augmenter considérablement leurs effectifs au début du XVIII ${ }^{\mathrm{e}}$ siècle, au point de compter en 1704 une douzaine d'incorporés, c'est-à-dire de membres à part entière, et une quarantaine d'agrégés. Il faut dire que le Calvaire était devenu, à cette époque, une véritable plaque tournante où l'on voyait se croiser les personnalités et les courants spirituels les plus divers, mais où par ailleurs, dominaient de plus en plus ìes ıdees chères aux discipies de Quesnel. Est-il besoin de rappeler que Charpentier, en son temps. avait été un ami de Saint-Cyran et que, depuis la fin du XVII ${ }^{e}$ siècle, la communauté du Mont-Valérien était pratiquement passée sous la domination de l'archevêque de Paris, le cardinal de Noailles, dont les sympathies pour le jansénisme n'étaient que trop connues. Aussi les prêtres du Calvaire allaient-ils se faire appelants en 1717 et persister dans cette attitude jusqu'à l'arrivée de Mgr Vintimille à la tête de l'archidiocèse de Paris en $1729^{7}$.

Vivant à proximité du célèbre pèlerinage, M. Varlet père ne pouvait éviter d'entretenir quelque relation avec les prêtres chargés de le desservir et, s'il faut en croire les Nouvelles Ecclésiastiques, c'est par son intermédiaire que Dominique-Marie fut mis en contact. dès 1699, sinon plus tôt, avec la communauté du Mont-Valérien ${ }^{8}$. Quoi qu'il en soit, le 6 octobre 1699, nous voyons le jeune Varlet se faire inscrire, à titre d'agrégé, dans la Congrégation de Messieurs les Prestres $d u$ Calvaire ${ }^{9}$. Pour autant que les documents officiels nous permettent de le savoir, il était encore de la société à la fin de l'année $1710^{10}$. C'est donc dire que durant au moins onze années le futur archevêque de Babylone fut en relation suivie avec la maison du Mont-Valérien et fut à même d'y recevoir les enseignements des chapelains qui, selon la formule on ne peut plus révélatrice des Nouvelles Ecclésiastiques, y répandaient depuis nombre d'années \& la bonne odeur de J.C. ${ }^{11}$ ».

Au moment de son agrégation, Dominique-Marie était déjà clerc, pour le compte du diocèse de Paris, et étudiant en théologie ${ }^{12}$. Il avait dû faire son entrée à Saint-Magloire l'année précédente tout en

7 La plupart de ces renseignements nous ont été aimablement fournis par M. Michel Guillot, des Archives Nationales de France, qui prépare actuellement un mémoire sur les ermites du Mont-Valérien.

8 Nouvelles Ecclésiastiques, loc. cit.

9 Cf. Registre des délibérations de la Congrégation de Mrs les Prestres du Calvaire du Mont-Valérien (1664-1744), Paris, Archives Nationales, LL 1591, pp. 38-39.

10 Ibid., pp. 115 et 117.

11 Nouvelles Ecclésiastiques, loc. cit.

12 Registre des délibérations..., p. 6. 
s'inscrivant aux cours du Collège de Navarre, puisque, à cette époque, l'enseignement, à Paris du moins, ne se donnait pas au séminaire, mais en faculté ${ }^{13}$, et c'est, par conséquent, dans la maison de Navarre que le jeune Varlet prépara, puis décrocha successivement son baccalauréat en théologie en 1701, sa licence, très probablement en 1704, et son doctorat en $1706^{14}$. Notons, au passage, que Saint-Magloire, dirigé par les Oratoriens, passait depuis un certain nombre d'années pour être un des principaux centres de diffusion des idées jansénistes à Paris ${ }^{15}$. C'est, en tout cas, dans cette maison que Varlet fera la connaissance de deux jansénistes notoires, Jacques Jubé (1674-1745), le futur liturgiste ${ }^{16}$, et Jean-Baptiste Paulin d'Aguesseau (1681-1738), le frère $\mathrm{du}$ chancelier ${ }^{17}$, avec lesquels il se liera d'une profonde et durable amitié.

Tout au long de ses années de formation, Dominique-Marie Varlet donne donc l'impression de vivre dans une atmosphère profondément janséniste et l'on est en droit de penser que, dès cette époque, il avait acquis l'essentiel de la mentalité qui, plus tard, allait le caractériser dans ses rapports avec Rome, celle d'un appelant irréductible, convaincu de la justesse de ses positions théologiques et du bien-fondé de ses droits. Prétendre, comme l'a fait Auguste Gosselin, et d'autres avant lui, que jusqu'en 1718, c'est-à-dire jusqu'à sa consécration épiscopale, Varlet n'avait rien laissé paraître de ses tendances jansénistes et qu'il n'était pas connu comme tel dans son entourage ${ }^{18}$ relève de la plus haute fantaisie, une fantaisie, soulignons-le, que contredit l'évidence même des faits.

\section{La vocation missionnaire}

L'année même où le collège de Navarre lui avait décerné son doctorat en théologie, soit en 1706, Varlet avait reçu l'ordination sacerdotale ${ }^{19}$ pour se voir presque aussitôt affecté au ministère paroissial dans les environs de Paris. Desservant d'Echarcon ${ }^{20}$, puis curé de Conflans, probablement dès $1710^{21}$, il n'hésitera pas à démissionner de ce dernier poste deux années plus tard, en raison d'un conflit l'opposant à certains religieux du voisinage, probablement les

13 A. DEGERT, Histoire des séminaires français jusqu'à la Révolution, t. II, Paris 1912, pp. 16-17.

14 Registre des délibérations..., pp. 55 et 96. Cf. aussi Nouvelles Ecclésiastiques, loc. cit.

15 A. DEGERT, op. cit., t. I, p. 190.

16 E. Precur, Les jansénistes du XVIII ${ }^{\bullet}$ siècle et la Constitution civile du Clergé, Paris 1929, p. 181.

17 Nouvelles Ecclésiastiques, loc. cit.

18 A. Gosselin, op. cit., p. 332.

19 Nouvelles Ecclésiastiques, loc. cit.

20 M. DELOCHE, art. cit., p. 43.

21 Registre des délibérations..., p. 115. 
bénédictins du prieuré de Sainte-Honorine, dépendant de l'abbé du Bec, qui, pour des raisons qui nous échappent, s'étaient mis en tête de faire échec aux initiatives de ce trop jeune et trop entıeprenant pasteur. Peut-être Varlet s'était-il lancé un peu trop rapidement dans des expériences liturgiques semblables à celles que pratiquait son ancien condisciple Jubé à Asnières ${ }^{22}$ ?

Quoi qu'il en soit, dépité par tant d'incompréhension et de mauvaise vôlunitié, le jeưinc curić cstima le moment venu de réaliser un rêve qui le hantait depuis déjà quelques années, celui de quitter la France et d'aller se consacrer outre-mer à l'évangélisation des infidèles. On est en droit de penser que cette vocation missionnaire datait de ses premières années d'études théologiques. Nous savons, par exemple, grâce à la correspondance du sous-principal du collège de Navarre, M. Delaissement ${ }^{23}$, qu'on s'intéressait beaucoup, à l'époque, dans cette maison, aux découvertes fabuleuses qu'étaient sensé avoir faites en Louisiane et dans le pays des Illinois les hommes de l'expédition de Cavelier de la Salle ${ }^{24}$. N'oublions pas que, quelques années auparavant. le célèbre $\mathrm{P}$. Hennepin avait fait paraître à Paris, puis à Utrecht, sa Description de la Louisiane (1683), sa Nouvelle Découverte d'un très grand pays (1697) et son Nouveau voyage (1698) qui avaient piqué. un peu partout, la curiosité d'un public européen de plus en plus sensible aux attraits de l'exotisme.

Mais il faut ajouter qu'au même collège de Navarre. on se passionnait également pour la question des rites chinois et qu'on suivait avec beaucoup d'attention le débat opposant les jésuites aux Messieurs des Missions-Étrangères à ce sujet. Inutile de dire de quel côté allaient les sympathies des professeurs et de leurs étudiants. En bénéficiait plus que tout autre $M$. de Lionne, évêque de Rosalie, qui. depuis son retour de Chine en 1702 n'avait cessé d'être en butte aux attaques des fils de saint Ignace ${ }^{25}$.

Fait significatif. en 1707, Dominique-Marie Varlet était entré lui-même en contact avec Artus de Lionne et s'était laissé progressivement acquérir à l'idée d'aller un jour se consacrer, selon le vœu de l'évêque de Rosalie, aux ouvres saintes abandonnées, c'est-à-dire aux missions étrangères. De plus en plus sensible à cet appel, Varlet était même allé se présenter en 1711 , alors qu'il était encore curé de Conflans, aux directeurs du Séminaire de la rue du Bac pour leur

22 Sur ces expériences de Jubé, cf. E. Preclin, op. cit., p. 181 et suiv.

23 Cette correspondance, adressée au P. Léonard, augustin déchaussé de Paris, couvre les années 1701-1705. Elle se trouve à la Bibliothèque Nationale de Paris, section des manuscrits, sous les cotes ms.fr. 19205 et 19213.

24 Delaissement au P. Léonard, 3 décembre 1701, ms.fr. 19205, fol. 29.

25 Le même au même, 4 et 9 novembre 1702, ibid., fol. 121.122. 
offrir ses services et demander à faire partie de leur société ${ }^{26}$. Avec sa démission de Conflans en 1712, cette offre devenait opérante et rien ne s'opposait plus à son départ pour un champ d'apostolat en pays étranger.

Or, justement, le Séminaire des Missions-Étrangères songeait à dépêcher de toute urgence de nouveaux effectifs aux établissements de Louisiane et des Illinois qui n'avaient reçu pratiquement aucun secours de Paris depuis 1707.

Ces territoires confiés aux prêtres du Séminaire de Québec par Mgr de Saint-Vallier avaient été évangélisés, à partir de 1698, par une pléiade d'hommes remarquables: François Jolliet de Montigny, Antoine Davion, les deux frères Buisson de St-Cosme, MM. Bergier, Boutteville, Foucault, mais le départ du premier pour la France en 1700, le massacre de l'abbé Foucault et de l'aîné des frères Buisson de SaintCosme par les Indiens en 1702 et 1706, la mort de M. Bergier aux Tamarois en 1707 n'avaient pas tardé à décimer les rangs d'une équipe par ailleurs trop réduite et débordée de travail, nécessitant du coup l'envoi de renforts importants. Or, surtout depuis l'incendie du Séminaire en 1701, Québec n'était plus tellement en mesure de faire face à de telles obligations ${ }^{27}$.

Sans doute, répondant à l'appel pressant de Mgr de Saint-Vallier, les directeurs de Paris avaient-ils réussi à envoyer à Mobile en 1704 MM. Henri Roulleaux de la Vente et Alexandre Huvé pour prendre la direction de la paroisse que l'évêque venait de confier à perpétuité aux Séminaires de Paris et de Québec. Mais M. de la Vente était repassé en France en 1710 et, n'eût été l'arrivée à Fort-Louis en 1707 d'un ancien vicaire de la paroisse Saint-Jacques de la Boucherie à Paris, M. le Maire, le contingent des prêtres des Missions-Étrangères aurait à peine suffi pour assurer le ministère auprès des Européens ${ }^{28}$.

Il faut ajouter que depuis les débuts de la guerre de Succession d'Espagne, les relations avec la Louisiane étaient devenues très difficiles et que, par ailleurs, la situation de la colonie du Mississippi, constamment en butte aux menaces des établissements anglais voisins, n'était guère encourageante. La suspension d'armes en Europe en 1712, valable pour les colonies à partir du mois d'août, puis la concession, un mois plus tard, d'un monopole commercial important sur toute la Louisiane

26 Nouvelles Ecclésiastiques, loc. cit.

27 A. Launay, op. cit., t. I, pp. 446-447; J. G. Shea, The Catholic Church in the Colonial Days, 1521-1763 (History of the Catholic Church in the United States), New York 1886, pp. 538-550; A. Gosselin, op. cit., pp. 167-173.

28 J. G. SHEA, op. cit., pp. 546-553. 
au grand financier Antoine Crozat ${ }^{29}$ allaient permettre à la colonie de respirer quelque peu, tout en lui inspirant à nouveau une certaine confiance en son avenir. Du coup le Séminaire de Paris pouvait songer à tenter un nouvel effort en faveur de la Louisiane.

Dominique-Marie Varlet fut donc désigné à l'automne de 1712 pour aller travailler à la relève des missions du Mississippi et de l'Illinois, mais plus particulièrement, du poste de Tamarois qui était resté pratiquement sans pasteur depuis la mort de M. Bergier en 1707.

Aussitôt décidé, presque aussitôt fait. Vers la fin de décembre, après avoir pris congé de sa vieille mère, l'ancien curé de Conflans était déjà en route pour Port-Louis, lieu de son embarquement ${ }^{30}$. Son frère, le futur procureur au Parlement, avait songé un moment à l'accompagner, arguant qu'il pourrait lui être d'un précieux secours dans son travail apostolique, mais Dominique-Marie, soupçonnant que ces pieux desseins pouvait fort bien couvrir de moins avouables ambitions, celle d'aller tenter fortune en Amérique, par exemple, réussit à le dissuader ${ }^{31}$ et, vers la fin de janvier, put enfin partir seul à destination de la Louisiane. La traversée, coupée d'un certain nombre d'escales aux Antilles, allait durer un peu plus de quatre mois.

Débarqué, le 6 juin, à Fort-Louis, à proximité de Mobile, Varlet nous apprend lui-même qu'il fut aussitôt atteint d'un flux de sang qui faillit l'emporter ${ }^{32}$, ce qui l'obligea à s'installer pour un temps sur place avec MM. Davion et le Maire en attendant que des circonstances plus favorables lui permissent de pousser jusqu'aux Illinois. Il allait, de fait, demeurer à Mobile jusqu'au début de 1715 .

\section{La vie missionnaire}

Les premières impressions recueillies par le nouveau missionnaire ne manquent pas d'intérêt. Le climat lui paraît sain, le sol, prometteur et, pour ce qui est des forêts, il ne manque pas de signaler à quel point elles sont giboyeuses, surtout au cours de la saison froide; mais il avoue, par contre, ne pas trouver le pays très beau, "car, dit-il, il n'est point encore défriché ${ }^{33}$ ». "C'est un pays sauvage, inculte ${ }^{34}$ ", écrit-il un peu plus tard, toujours dans le même sens. Et encore: " Vous voyez que ce pays, sur la description que je vous en fais qui

29 Cf. Edit du Roi portant l'établissement de la Louisiane par le Sieur Crozat, du 14 septembre 1712, dans Edits, Ordonnances royaux, déclarations du Conseil d'Etat du Roi concernant le Canada, t. I, Québec 1854, pp. 327-331.

30 Varlet à sa mère, Paris, 18 décembre 1712, cité par M. Deloche, art. cit., pp. $43-44$.

31 Le même à son frère, Fort-Louis, 16 janvier 1713, ibid., pp. 45-46.

32 Le même à sa mère, Fort-Louis, 13 juillet 1713 , $i$ bid., p. 46.

33 Ibid.

34 Le même à son frère, Fort-Louis, [s.d.], ibid., p. 52. 
est naturelle, n'est point tentatif [sic] et que ça n'est pas comme on le croit en France, une merveille du monde ${ }^{35}$. "

Ce dernier trait de mentalité mérite d'être souligné, car il contraste singulièrement, nous semble-t-il, avec les courants qui se font de plus en plus jour, à l'époque, en Europe, à la faveur des récits de voyage et des descriptions du Nouveau Monde: mythe du bon sauvage, sentiment de la nature, goût de l'exotisme, pour ne mentionner que ceux-là, tous repères appelés à prendre de plus en plus d'importance sur la carte idéologique des $\mathrm{XVIII}^{\bullet}$ et $\mathrm{XIX}^{\bullet}$ siècles ${ }^{36}$.

En ce qui concerne le travail missionnaire comme tel, Varlet a tôt fait de constater que les effectifs sur place, loin d'être à la hauteur des besoins, suffisent à peine à assurer le service des colons européens et des quelques Indiens établis à proximité de Mobile et de Fort-Louis. Par ailleurs, ses premiers contacts avec la culture, les mœurs et les coutumes indigènes lui permettent de soupçonner, dès l'abord, les difficultés inhérentes à tout travail d'évangélisation auprès de peuplades simples, certes, mais combien frustres et grossières. Il se rend surtout très bien compte, en théologien chevronné qu'il est, de la quasiimpossibilité d'exprimer dans des dialectes parfois très pauvres certains des éléments pourtant essentiels du donné révélé. Autant d'obstacles qui le gênent dans l'accomplissement de sa tâche missionnaire et qui, personnellement, - il ne s'en cache pas - le font beaucoup souffrir ${ }^{37}$.

Mais Varlet n'en était encore là qu'à ses premières armes. Au début de 1715, alors qu'il venait d'être nommé vicaire général de l'évêque de Québec pour les missions du Mississippi et de l'Illinois, il apprit que La Motte-Cadillac, gouverneur de la Louisiane, organisait une expédition vers le nord dans le but d'aller vérifier l'existence de mines d'argent que certains prétendaient $\mathrm{y}$ avoir aperçues. Il décida donc de profiter de l'occasion pour quitter enfin Mobile et aller s'installer à Tamarois où, selon les directives qui lui avaient été primitivement données à Paris, il devait travailler à la poursuite de l'œuvre entreprise par M. Bergier ${ }^{38}$.

Tamarois ou Cahokia, située un peu à l'ouest de l'actuelle ville de Saint-Louis, comptait alors, outre son contingent d'indigènes, les Tamarois, tribu illinoise apparentée aux Algonquins, une cinquantaine de familles canadiennes ${ }^{39}$. L'établissement jouissait depuis peu d'une

$35 \quad$ Ibid., p. 53.

36 P. HAZARD, La crise de la conscience européenne (1680-1715), t. I, pp. 16-18.

37 Varlet à sa mère, Fort-Louis, 23 novembre 1713 , cité par M. Deloche, art. cit., pp. 47-48; le même à la même, Fort-Louis, 7 avril 1714, ibid., p. 48; le même à son frère, [s.d.], ibid., pp. 48-52.

38 M. DELOCHE, art. cit., p. 53.

39 J. G. SheA, op. cit., p. 554. 
tranquillité relative, fruit de la paix que les autorités coloniales venaient de signer avec la tribu rivale des Renards ${ }^{40}$. A tout considérer, Varlet n'avait pas à regretter Mobile et Fort-Louis, bien que, pour autant que nous puissions le soupçonner, sa nouvelle tâche missionnaire ne dut pas lui paraître beaucoup plus facile ni plus consolante que celle qui l'avait précédée.

Les Illinois, en effet, n'avaient pas très bonne réputation. S'il faut en croire le témoignage des pères jésuites qui, les premiers. avaient travaillé à leur conversion, ils étaient non seulement « lâches, traîtres. légers et inconstans", mais également "fourbes [...], brutaux, sans d'honneur». Susceptibles de tout faire quand on se montrait libéral à leur égard, ils pouvaient, en d'autres circonstances, être de la pire ingratitude "et sans reconnaissance" aucune. Au sentiment de la plupart des missionnaires, il était difficile de trouver peuple plus immoral - la polygamie y était fréquente - ni plus superstitieux. Sans doute les Illinois se montraient-ils habituellement très hospitaliers et très bienveillants envers les Européens et, plus particulièrement, envers les robes noires, mais cela ne pouvait faire oublier, encore moins compenser des vices qui, justement, constituaient le principal obstacle à la pénétration chez eux du christianisme ${ }^{41}$. La mission confiée à Varlet n'en était donc pas une de tout repos.

Malheureusement, nous sommes assez mal renseignés sur les détails de son ministère auprès des habitants de Cahokia. Les quelques lettres écrites à sa famille durant cette période ne nous apprennent. pour ainsi dire, rien, si ce n'est que Varlet donnait le meilleur de son temps à ses Tamarois, n'hésitant pas à les accompagner. chaque hiver, lorsqu'ils partaient pour leurs territoires de chasse ${ }^{42}$. Il faut dire que son séjour aux Illinois fut d'assez courte durée.

Dès le printemps de 1717 , en effet, nous le voyons reprendre l'aviron, cette fois en direction de Québec ${ }^{43}$. Les besoins grandissants de sa mission, mais, peut-être, plus encore, le souci de consolider sa position face aux revendications des jésuites qui continuaient à déplorer la présence de prêtres du Séminaire sur un territoire qu'ils estimaient leur avoir été réservé, rendaient impérieux ce voyage qui devait permettre non seulement de recruter un certain nombre d'auxiliaires, mais aussi d'obtenir de Mgr de Saint-Vallier la confirmation des lettres patentes de 1698.

40 Varlet à son frère, Tamaroas, 2 novembre 1716, cité par M. Deloche. art. cit., p. 54 .

41 Cf. la lettre du P. Gabriel Marest (9 novembre 1712), dans Lettres édifiantes et curieuses, t. VI, Paris 1781 , p. 320 et suiv.

42 Varlet à sa mère, Tamaroas, 3 novembre 1716 , cité par V. Deloche, art. cit., p. 55 .

43 Le même à son frère, Tamaroas, 2 mars 1717, ibid. 
Varlet nous a laissé un récit détaillé de son voyage. Parti le 24 mars de Cahokia, il ne réussit à rallier le poste de Michillimakinac. première étape importante de son périple, que deux mois plus tard, soit le 20 juin, et cela, après une exténuante remontée de la rivière Illinois et la traversée de l'immense lac du même nom ${ }^{44}$. Nouveau départ, huit jours plus tard, cette fois en direction de Détroit, par voie du lac Huron, puis, vers la fin de juillet, embarquement à destination de Montréal que notre missionnaire allait atteindre le 2 septembre, après avoir franchi deux importantes nappes d'eau, les lacs Erié et Frontenac ${ }^{45}$, et admiré au passage la chute du Niagara, presque aussi haute, écrit Varlet à sa mère, que les tours de Notre-Dame de Paris. Neuf jours plus tard, soit le 11 septembre, il était à Québec ${ }^{46}$. Son voyage avait duré, en tout et partout, un peu moins de six mois.

Dès le 6 octobre, nous le voyons obtenir de Mgr de Saint-Vallier de nouvelles lettres patentes confirmant les privilèges déjà obtenus par le Séminaire de Québec pour la mission de Tamarois avec défense à tout autre corps - il s'agit évidemment des jésuites - de tenter quoi que soit à l'encontre des droits du Séminaire ${ }^{47}$. Restait à régler le problème des effectifs. Mettant à profit les longs mois d'hiver, Varlet multiplia les démarches et fit si bien qu'il réussit finalement à convaincre ses collègues de laisser le chanoine Calvarin et M. Thaumur de la Source retourner avec lui à Cahokia ${ }^{48}$.

Notons toutefois qu'en septembre 1718, Varlet et ses compagnons n'avaient pas encore quitté Québec, ce qui contredit nettement l'affirmation d'Auguste Gosselin selon laquelle Varlet n'aurait fait que passer dans la colonie et se serait hâté de rentrer dans sa mission après être resté "à peine quelques jours " au Séminaire ${ }^{49}$. Varlet fut, en réalité. près de treize mois dans la capitale.

Malheureusement, mise à part une courte lettre à sa mère, il ne nous donne que très peu de renseignements sur son séjour dans la ville épiscopale et les fonds d'archives locaux, pour étonnant que cela puisse paraître, ne nous fournissent guère plus de détails. Serait-ce que sa présence à Québec passa inaperçue ou qu'elle fit si peu de bruit que les chroniqueurs de l'époque ne songèrent même pas à en faire état? Ou ne serait-ce pas plutôt que, soucieux de la bonne réputation de pp. $56-57$.

Aujourd'hui Michigan.

Aujourd'hui Ontario.

46 Varlet à sa mère, Québec, 16 octobre 1717, cité par M. Deloche, art. cit.,

${ }_{47}$ Le texte de ces lettres, datées du 6 octobre 1717, dans Mandements des évêques de Québec, p.p. H. TÊTU et C. O. GaGnon, vol. I, Québec 1887, pp. 495-496.

48 A. Gosselin, op. cit., p. 332.

49 Ibid., pp. 332; 333-334. 
l'Église de Nouvelle-France, certaines mains pieuses, apprenant plus tard que Varlet était devenu schismatique, prirent soin d'effacer la plupart des traces de son séjour en terre canadienne? Nous ne le saurons peut-être jamais. Quoi qu'il en soit, il serait surprenant que le futur archevêque de Babylone n'ait pas eu l'occasion, en treize mois, d'entrer en contact avec les maisons religieuses de la capitale et n'ait pas réussi à leur communiquer un peu de cet esprit qui allait bientôt servir à le faire connaître à toute l'Europe.

A la fin de septembre 1718, alors qu'il s'apprêtait à partir pour les Illinois, Varlet reçut de ses supérieurs de Paris une lettre lui ordonnant de rentrer le plus tôt possible en France. Il laissa donc ses collègues Calvarin et Thaumur de la Source partir seuls pour le pays des Tamarois et, sans plus tarder, s'embarqua, vers le début d'octobre très probablement, à destination de la métropole ${ }^{50}$. Le 13 novembre, il était à La Rochelle "1, puis, quinze jours plus tard, à Paris où il apprit sa nomination comme coadjuteur de l'archevêque de Babylone, M. de Saintulong ${ }^{52}$.

L'aventure américaine était terminée; une autre commençait qui allait le conduire sous d'autres cieux et vers d'autres horizons. Il serait difficile de dire dans quelle mesure la première l'avait préparé à la seconde, mais, chose certaine, cette dernière allait lui causer des souffrances et des ennuis autrement plus cruels que ceux qu'il avait connus en Amérique. Aussi ne faut-il pas nous surprendre de le voir écrire en 1733, au plus fort de ses démêlés avec Rome: "Je regrette encore souvent les bois de l'Amérique ${ }^{53}$. Peut-être eût-il mieux valu pour lui qu'il ne les eût jamais quittés.

Pierre Hurtubise, o.m.i.

Professeur d'histoire ecclésiastique, Université Saint-Paul, Ottawa.

50 Un de ses compagnons de traversée fut peut-être Dom Georges-François Poulet, bénédictin janséniste réfugié au Canada depuis 1714 et qui, d'après Gosselin (op. cit., p. 330), serait rentré en France, le 2 octobre 1718 , à bord du Mutine.

51 Varlet à sa mère, La Rochelle, 13 novembre 1718, cité par M. Desoche, art. cit., p. 58.

52 D'après une note manuscrite sur la lettre précédente.

53 M. DELOCHE, art. cit., p. 59. 